University of Nebraska - Lincoln

DigitalCommons@University of Nebraska - Lincoln

2-1-1993

\title{
Nonlinear analysis of relativistic harmonic generation by intenselasers in plasmas
}

\author{
Eric Esarey \\ US Naval Res. Lab., Washington, DC, ehesarey@lbl.gov
}

A. Ting

US Naval Res. Lab., Washington, DC

P. Sprangle

US Naval Res. Lab., Washington, DC

Donald Umstadter

University of Nebraska-Lincoln, donald.umstadter@unl.edu

X. Liu

US Naval Res. Lab., Washington, DC

Follow this and additional works at: https://digitalcommons.unl.edu/physicsumstadter

Part of the Physics Commons

Esarey, Eric; Ting, A.; Sprangle, P.; Umstadter, Donald; and Liu, X., "Nonlinear analysis of relativistic harmonic generation by intenselasers in plasmas" (1993). Donald Umstadter Publications. 15.

https://digitalcommons.unl.edu/physicsumstadter/15

This Article is brought to you for free and open access by the Research Papers in Physics and Astronomy at DigitalCommons@University of Nebraska - Lincoln. It has been accepted for inclusion in Donald Umstadter Publications by an authorized administrator of DigitalCommons@University of Nebraska - Lincoln. 


\title{
Nonlinear Analysis of Relativistic Harmonic Generation by Intense Lasers in Plasmas
}

\author{
E. Esarey, A. Ting, P. Sprangle, D. Umstadter, and X. Liu
}

\begin{abstract}
A linearly polarized, ultra-intense laser field induces transverse plasma currents which are highly relativistic and nonlinear, resulting in the generation of coherent harmonic radiation in the forward direction (i.e., co-propagating with the incident laser field). A nonlinear cold fluid model, valid for ultrahigh intensities, is formulated and used to analyze relativistic harmonic generation. The plasma density response is included self-consistently and is shown to significantly reduce the current driving the harmonic radiation. Phase detuning severely limits the growth of the harmonic radiation. The effects of diffraction are considered in the mildly relativistic limit. No third harmonic signal will emerge from a uniform plasma of near infinite extent. A finite third harmonic signal requires the use of a semi-infinite or finite slab plasma. For an initially uniform plasma, no second harmonic radiation is generated. Generation of even harmonics requires transverse gradients in the initial plasma density profile.
\end{abstract}

\section{INTRODUCTION}

$\mathbf{T}$ HE interaction of ultra-intense laser pulses in plasmas is rich in a variety of phenomena [1]-[22]. These phenomena have gained particular relevance due to the development of compact lasers which produce ultrashort pulses $(\leq 1 \mathrm{ps})$ at ultrahigh powers $(\geq 1 \mathrm{TW})$ and intensities $\left(\geq 10^{18} \mathrm{~W} / \mathrm{cm}^{2}\right)[2]$, [23]. For example, the production of harmonic radiation may occur by several mechanisms. At modest intensities, lasers interacting with neutral gases have been observed to produce coherent harmonic radiation at well past the $53^{\text {st }}$ harmonic due to atomic effects [13]. At ultrahigh intensities, a gas is readily ionized and the effects of the free plasma electrons become exceedingly important. The ionization process itself results in electron currents which can produce harmonic radiation [14], [15]. In a fully ionized plasma, harmonics can be produced by i) relativistic harmonic generation in the forward direction (the propagation direction of the incident laser) [1], [8], [17]-[20], ii) stimulated backscattered harmonic generation [1], [21], and iii) incoherently, by nonlinear Thomson scattering [1], [22]. Recent experiments on these processes have also been performed [2], [15]-[18]. This paper discusses the details of process i), relativistic harmonic generation in fully ionized plasmas. The growth, dephasing, and saturation of the harmonics will be analyzed.

Manuscript received May 21, 1992; revised October 6, 1992. This work was supported by the Office of Naval Research, the Department of Energy and the National Science Foundation Center for Ultrafast Optical Science.

E. Esarey, A. Ting, and P. Sprangle are with the Beam Physics Branch, Plasma Physics Division, Naval Research Laboratory, Washington, DC 20375.

D. Umstadter and X. Liu are with the Center for Ultrafast Optical Science, University of Michigan, Ann Arbor, MI 48109.

IEEE Log Number 9206345.

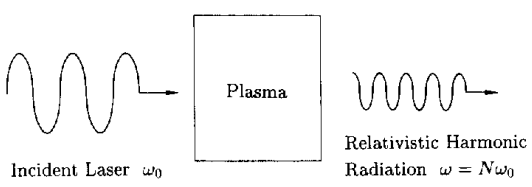

Fig. 1. Schematic of an intense laser interacting with a plasma to produce coherent relativistic harmonic radiation in the forward direction.

An intense laser field interacting with a plasma induces transverse currents associated with the quiver motion of the electrons. For ultrahigh intensities and linear polarizations, the induced plasma current becomes highly relativistic and nonlinear, resulting in the generation of coherent harmonic radiation in the forward direction (see Fig. 1). The transverse quiver momentum, $\mathbf{p}_{q}$, of an electron in a 1-D laser field is given by $\mathbf{p}_{q}=m_{0} c \mathbf{a}_{0}$, where $\mathbf{a}_{0}=e \mathbf{A}_{0} / m_{0} c^{2}$ is the normalized vector potential of the incident laser field. The quiver velocity, $\mathbf{v}_{q}$, is given by $\mathbf{v}_{q}=c \mathbf{a}_{0} / \gamma_{\perp}$, where $\gamma_{\perp} \simeq\left(1+a_{0}^{2}\right)^{1 / 2}$ is the relativistic factor associated with the transverse electron motion. Consider a linearly polarized incident laser field of the form $\mathbf{a}_{0}(z, t)=\hat{a}_{0} \cos \theta_{0} \mathbf{e}_{x}$, where $\theta_{0}=k_{0} z-\omega_{0} t$ and $k_{0}$ and $\omega_{0}$ are the wavenumber and frequency of the incident laser field, respectively. In the mildly relativistic limit, $\hat{a}_{0}^{2} \ll 1$, the quiver velocity is sinusoidal. At ultrahigh intensities, $\hat{a}_{0}^{2} \geq 1$, the quiver velocity contains a spectrum of harmonic components. This nonlinear electron quiver motion leads to the generation of relativistic harmonic radiation [1], [8], [17]-[20]. The laser strength parameter, $\hat{a}_{0}$, is related to the intensity, $I_{0}$, of the incident laser field by

$$
\hat{a}_{0} \simeq 8.5 \times 10^{-10} \lambda_{0}[\mu \mathrm{m}] I_{0}^{1 / 2}\left[\mathrm{~W} / \mathrm{cm}^{2}\right]
$$

and to the laser power, $P_{0}$, by $P_{0}[\mathrm{GW}] \simeq 21.5\left(\hat{a}_{0} r_{0} / \lambda_{0}\right)^{2}$, where $r_{0}$ is the spot size of the Gaussian transverse profile and $\lambda_{0}=2 \pi / k_{0}$ is the incident laser wavelength. For $\lambda_{0} \simeq$ $1 \mu \mathrm{m}$, ultrahigh intensities $I_{0} \gtrsim 10^{18} \mathrm{~W} / \mathrm{cm}^{2}$ imply $\hat{a}_{0}^{2} \gtrsim 1$ and, hence, highly nonlinear and relativistic electron motion. Such intensities are currently available from compact laser systems based on the method of chirped-pulse amplification [2], [23].

Relativistic harmonic generation was first described and analyzed by Sprangle, et al. [8]. In [8], the independent variables $\zeta=z-c t$ and $\tau=t$ were used along with a quasistatic plasma response. Expressions were derived for the growth of the harmonic radiation in the linear regime in which the harmonic field amplitude is proportional to the laser-plasma interaction distance, $L$. These expressions are qualitatively valid for interaction distances less than the phase 
detuning length, $c \tau_{d}$. Use of the variables $\zeta, \tau$ along with the quasistatic approximation is not adequate to accurately describe saturation of the relativistic harmonics by phase detuning. More recently, by using the variables $\xi=z-v_{p h} t$ and $\tau=t$, where $v_{p h}$ is the phase velocity of the incident laser field, along with the quasistatic plasma response, the authors accurately analyzed the saturation of the relativistic harmonics by phase detuning [1]. Independent analyses of saturation of the third harmonic by phase detuning have also been recently performed [19], [20].

This paper is organized as follows. The remainder of the introduction describes the basic physics of third harmonic generation using a simplified 1-D model in the mildly relativistic limit, $\hat{a}_{0}^{2} \ll 1$. The importance of collective, space-charge, and detuning effects are discussed. It is shown that the self-consistent plasma density response significantly reduces the source current driving the harmonic radiation. Phase detuning places a severe limit on the growth of the harmonic radiation. In Section II, a 1-D nonlinear model valid for ultrahigh intensities, $\hat{a}_{0}^{2} \geq 1$, is formulated and used to study the generation of coherent radiation at odd harmonics. A general expression for the nonlinear index of refraction and the dispersion relation for a laser field in the limit $\hat{a}_{0}^{2} \geq 1$ are presented. The collective plasma response is included selfconsistently and the saturation amplitude of the harmonics by phase detuning is calculated. This is done in the long pulse limit, $c \tau_{L} \gg \lambda_{p}$, where $\tau_{L}$ is the incident laser pulse duration and $\lambda_{p}=2 \pi c / \omega_{p}$, where $\omega_{p}$ is the electron plasma frequency. For a $\tau_{L}=1 \mathrm{psec}$ laser pulse, $c \tau_{L} \gg \lambda_{p}$ implies $n_{0} \gg 10^{16}$ $\mathrm{cm}^{-3}$, where $n_{0}$ is the ambient plasma density. In Section III, the effects of diffractive spreading of the radiation fields are determined using a 3-D model in the mildly relativistic limit, $\hat{a}_{0}^{2} \ll 1$. Third harmonic generation from semi-infinite and finite slab plasmas are analyzed. The effects of transverse gradients in the initial plasma density are discussed, which lead to the generation of radiation at even harmonics. A conclusion is presented in Section IV.

\section{A. Quiver Model, Collective Effects and Detuning}

The process by which relativistic effects produce coherent harmonic radiation may be understood by considering a simplified "quiver" model, which includes only the effects of the relativistic electron quiver motion. Other effects, such as the plasma density response, will be discussed below. A linearly polarized laser field will be assumed, $a_{0}(z, t)=\hat{a}_{0} \cos \theta_{0}$. In the quiver model, the transverse plasma current is $J_{q}=$ $-e n_{0} v_{q}$, where $v_{q}$ is the relativistic electron quiver velocity (discussed above) and $n_{0}$ is the ambient plasma density. The quiver current $J_{q}$ acts as the source term in the wave equation which drives the harmonic radiation, $\left(\nabla^{2}-\partial^{2} / c^{2} \partial t^{2}\right) a=S_{q}$, where

$$
S_{q} \simeq k_{p}^{2} \hat{a}_{0} \cos \theta_{0}\left(1+\hat{a}_{0}^{2} \cos ^{2} \theta_{0}\right)^{-1 / 2}
$$

and $\omega_{p}=c k_{p}=\left(4 \pi e^{2} n_{0} / m\right)^{1 / 2}$ is the plasma frequency. In the limit $\hat{a}_{0}^{2} \ll 1$, the denominator in (2) may be expanded and the component driving the $N^{t h}$ odd harmonic $\left(\omega_{N}=N \omega_{0}\right)$ may be determined. For example, the ratio of the third harmonic power to the fundamental is $P_{3} / P_{0} \simeq$ $\left(\hat{a}_{0}^{2} k_{p}^{2} L / 16 k_{0}\right)^{2} \equiv R_{q}$, where $L$ is the laser-plasma interaction length and $L<L_{d}$ has been assumed, where $L_{d}$ is the detuning distance (discussed below). Hence, in the quiver limit, $P_{3} \sim L^{2} n_{0}^{2} I_{0}^{3}$.

The quiver model assumed that the plasma response is dominated by the electron quiver motion. This is an oversimplification and collective plasma effects, i.e., the plasma density response, cannot be neglected. Including the density response, $\delta n(z, t)$, in the transverse current gives $J_{\perp}=$ $-e\left(n_{0}+\delta n\right) v_{\perp}$. Letting $v_{\perp}=v_{q}$ gives $S=k_{p}^{2} a_{0}(1+$ $\left.\delta n / n_{0}-a_{0}^{2} / 2\right)$, where the term $a_{0}^{2} / 2$ arises from expanding the relativistic factor, assuming $a_{0}^{2} \ll 1$. Using 1-D cold fluid theory, the density response $\delta n$ may be calculated, giving

$$
S \simeq k_{p}^{2} \hat{a}_{0} \cos \theta_{0}\left(1-\frac{\hat{a}_{0}^{2}}{4}-\frac{3}{16} \frac{\omega_{p}^{2}}{\omega_{0}^{2}} \hat{a}_{0}^{2} \cos 2 \theta_{0}\right) .
$$

Hence, the effect of including the density response is to reduce [7], [8] the source term for the third harmonic, $\sim \exp \left( \pm 3 i \theta_{0}\right)$, by the factor $3 \omega_{p}^{2} / 4 \omega_{0}^{2} \ll 1$ as compared to the quiver model. The power in the third harmonic will be reduced by the square of this factor, $P_{3} / P_{0}=R_{q}\left(3 \omega_{p}^{2} / 4 \omega_{0}^{2}\right)^{2}$. Hence, in the 1-D limit, $P_{3} \sim L^{2} n_{0}^{4} I_{0}^{3}$. Physically, this reduction arises from the longitudinal ponderomotive force, $F_{z} \sim-\nabla_{z} a_{0}^{2} \sim k_{0} \hat{a}_{0}^{2} \sin 2 \theta_{0}$. This modulates the density, $\delta n / n_{0} \sim \exp \left( \pm 2 i \theta_{0}\right)$, in such a way that it nearly cancels (to order $\omega_{p}^{2} / \omega_{0}^{2}$ ) the contribution from the relativistic factor, $a_{0}^{2} / 2$. In the absence of the space charge potential, $\omega_{p}^{2} \rightarrow 0$, this cancellation is exact and no third harmonics are generated in the 1-D limit.

The harmonic radiation will reach a maximum amplitude after a detuning distance [1], [19], [20], $L=L_{d}$. The phase velocity $v_{p}$ of an electromagnetic wave of frequency $\omega$ in a plasma is given by $v_{p} / c \simeq 1+\omega_{p}^{2} / 2 \omega^{2}$, where $\omega_{p}^{2} / \omega^{2} \ll 1$ and $a_{0}^{2} \ll 1$ have been assumed. The phase velocity of the incident laser, $\omega=\omega_{0}$, is greater than that of the third harmonic, $\omega=3 \omega_{0}$. Hence, the third harmonic, which is being driven by the incident laser, eventually becomes out of phase with the incident laser. The maximum amplitude of the third harmonic occurs after the detuning distance defined by $L_{d} \Delta v_{p} / c=$ $\lambda_{3} / 2$, where $\Delta v_{p}=4 c \omega_{p}^{2} / 9 \omega_{0}^{2}$ is the difference in the phase velocities of the incident and third harmonic fields and $\lambda_{3} \simeq$ $\lambda_{0} / 3$ is the wavelength of the third harmonic. Hence, $L_{d} \simeq$ $3 \lambda_{p}^{2} / 8 \lambda_{0}$, where $\lambda_{p}=2 \pi / k_{p}$ is the plasma wavelength. The maximum amplitude of the third harmonic power at saturation may be estimated by setting $L=L_{d}$, giving $P_{3} / P_{0} \simeq$ $\left(\hat{a}_{0} \lambda_{0} / \lambda_{p}\right)^{4} \%$. As an example, consider a $\lambda_{0}=1 \mu \mathrm{m}$ laser with $I_{0}=5 \times 10^{17} \mathrm{~W} / \mathrm{cm}^{2}\left(\hat{\mathrm{a}}_{0} \simeq 0.6\right)$ and a plasma of density $\mathrm{n}_{0}=$ $10^{19} \mathrm{~cm}^{-3}\left(\lambda_{\mathrm{p}} \simeq 11 \mu \mathrm{m}\right)$. The third harmonic power is given by $P_{3} / P_{0} \simeq 9 \times 10^{-8}$. The detuning length is prohibitively short, $L_{d} \simeq 45 \mu \mathrm{m}$. The third harmonic pulse length is approximately equal to that of the incident laser pulse.

\section{NONLINEAR FORMULATION}

The 1-D fields associated with the laser-plasma interaction can be described by the normalized transverse vector potential, 
$\mathbf{a}(z, t)=e \mathbf{A}_{\perp}(z, t) / m_{0} c^{2}$, and the normalized scaler potential, $\phi(z, t)=e \Phi(z, t) / m_{0} c^{2}$. Coulomb gauge will be used, $\nabla \cdot \mathbf{A}=0$, which implies $A_{z}=0$. It is convenient to introduce the independent variables $\xi=z-c \beta_{t} t$ and $\tau=t$, where $\beta_{t}$ is the normalized transform velocity which will be specified below. Using the $\xi, \tau$ variables, the normalized potentials a and $\phi$ satisfy the 1-D wave equation and Poisson's equation, which are given by

$$
\begin{gathered}
\left(\frac{1}{\gamma_{t}^{2}} \frac{\partial^{2}}{\partial \xi^{2}}+\frac{2 \beta_{t}}{c} \frac{\partial^{2}}{\partial \xi \partial \tau}-\frac{1}{c^{2}} \frac{\partial^{2}}{\partial \tau^{2}}\right) \mathbf{a}=k_{p}^{2} \frac{n}{\gamma n_{0}} \mathbf{a} \\
\frac{\partial^{2}}{\partial \xi^{2}} \phi=k_{p}^{2}\left(\frac{n}{n_{0}}-1\right)
\end{gathered}
$$

where $\gamma_{t}^{-2}=1-\beta_{t}^{2}, \omega_{p}=c k_{p}=\left(4 \pi e^{2} n_{0} / m_{0}\right)^{1 / 2}$ is the ambient plasma frequency, $n(\xi, \tau)$ is the plasma electron density, $n_{0}$ is the ambient density, $\gamma(\xi, \tau)=\left(1-\beta^{2}\right)^{-1 / 2}$ is the relativistic factor associated with the plasma electrons and $\beta(\xi, \tau)=v / c$ is the normalized electron fluid velocity. In deriving the right side of (4), use was made of the fact that the transverse canonical momentum is invariant and that prior to the laser interaction the plasma is assumed to be stationary, i.e., $\beta_{\perp}=a / \gamma$. Also, the ions are assumed to be stationary.

The electron fluid quantities $n, \beta_{z}$ and $\gamma$ are assumed to satisfy the cold, relativistic fluid equations. Using the $\xi, \tau$ variables, the continuity equation and the axial momentum equation may be written as

$$
\begin{gathered}
\frac{\partial}{\partial \xi}\left[n\left(\beta_{t}-\beta_{z}\right)\right]=\frac{1}{c} \frac{\partial}{\partial \tau} n \\
\frac{\partial}{\partial \xi}\left[\gamma\left(1-\beta_{t} \beta_{z}\right)-\phi\right]=-\frac{1}{c} \frac{\partial}{\partial \tau}\left(\gamma \beta_{z}\right)
\end{gathered}
$$

respectively. Equations (4)-(7), together with $\gamma=(1+$ $\left.a^{2}\right)^{1 / 2} /\left(1-\beta_{z}^{2}\right)^{1 / 2}$, form a complete set of fully nonlinear, relativistic cold fluid equations which describe the 1-D laser-plasma interaction. The 1-D assumption is valid provided that the radiation spot size $r_{s}$ is large compared to the plasma wavelength $\lambda_{p}=2 \pi / k_{p}$, i.e., $r_{s} \gg \lambda_{p}$. The cold fluid assumption is valid provided that i) the electron quiver velocity is much greater than the transverse thermal velocity and ii) the phase velocity of the driven plasma oscillations is much greater than the axial thermal velocity, as is the case in the following.

The primary focus of this section is to determine the coherent harmonic radiation excited by a long pulse incident laser field, $c \tau_{L} \gg \lambda_{p}$. To study coherent harmonic radiation, it is convenient to set the transform velocity, $\beta_{t}$, equal to the phase velocity, $\beta_{p h}$, of the incident laser field, $\beta_{t}=\beta_{p h}$. The incident laser field is of the form $a_{0}=\hat{a}_{0} \cos k_{0} \xi$, where $k_{0} \xi=k_{0}\left(z-c \beta_{p h} t\right), \beta_{p h}=\omega_{0} / c k_{0}$ is the normalized phase velocity, $\omega_{0}$ is the frequency, and $k_{0}$ is the wavenumber of the pump laser field. The pump laser amplitude is slowly varying, $\left|\partial \hat{a}_{0} / \partial \xi\right| \sim \hat{a}_{0} / c \tau_{L}$, and is assumed to be independent of $\tau$, i.e., pump depletion effects are neglected. The effects of diffraction are consider in Section III. In determining the plasma response to an incident laser field of the form $a_{0}=a_{0}(\xi)$, the $\tau$ derivatives are dropped in (6)-(7). Strictly speaking, for a general laser field of the form $a_{0}=a_{0}(\xi, \tau)$, the $\tau$ derivatives may be neglected provided that the transit time of the electrons through the laser pulse, $\tau_{L}$ (= laser pulse duration), is small compared to the evolution time, $\tau_{E}$, of the laser envelope, $\tau_{L} \ll \tau_{E}$, i.e., the quasistatic approximation [8]. Assuming a quasistatic plasma response, (6)-(7) imply the existence of two constants of the motion,

$$
\begin{aligned}
n\left(\beta_{t}-\beta_{z}\right) & =\beta_{t} n_{0}, \\
\gamma\left(1-\beta_{t} \beta_{z}\right)-\phi & =1 .
\end{aligned}
$$

Equations (8a)-(b) may be solved to give expressions for the fluid quantities $n, \beta_{z}$ and $\gamma$ in terms of the fields $a$ and $\phi$,

$$
\begin{aligned}
n / n_{0} & =\gamma_{t}^{2} \beta_{t}\left[\left(1-1 / \psi^{2} \gamma_{t}^{2}\right)^{-1 / 2}-\beta_{t}\right], \\
\beta_{z} & =\frac{\beta_{t}-\left(1-1 / \psi^{2} \gamma_{t}^{2}\right)^{1 / 2}}{1-\beta_{t}\left(1-1 / \psi^{2} \gamma_{t}^{2}\right)^{1 / 2}}, \\
\gamma & =\gamma_{t}^{2}(1+\phi)\left[1-\beta_{t}\left(1-1 / \psi^{2} \gamma_{t}^{2}\right)^{1 / 2}\right], \\
n / \gamma n_{0} & =\beta_{t}(1+\phi)^{-1}\left(1-1 / \psi^{2} \gamma_{t}^{2}\right)^{-1 / 2}
\end{aligned}
$$

where $\psi^{2}=(1+\phi)^{2} /\left(1+a^{2}\right)$. Notice that for the case $\beta_{t}=\beta_{p h}, \gamma_{t}^{-2}=\gamma_{p h}^{-2}<0$, since $\beta_{p h}^{2}>1$ for a radiation field in a plasma.

Using the above expressions for the fluid quantities, expressions for the normalized transverse plasma current, $k_{p}^{2} n_{\mathrm{a}} / n_{0} \gamma$, and the normalized charge perturbation, $k_{p}^{2}\left(n / n_{0}-1\right)$, can be found and inserted into the wave equation and Poisson's equation (4)-(5). This results in two coupled nonlinear equations for the potentials $a$ and $\phi$,

$$
\begin{aligned}
\left(\frac{1}{\gamma_{t}^{2}} \frac{\partial^{2}}{\partial \xi^{2}}+\frac{2 \beta_{t}}{c} \frac{\partial^{2}}{\partial \xi \partial \tau}\right. & \left.-\frac{1}{c^{2}} \frac{\partial^{2}}{\partial \tau^{2}}\right) \mathbf{a} \\
= & \frac{k_{p}^{2}}{(1+\phi)}\left(1+\frac{1}{k_{p}^{2} \gamma_{t}^{2}} \frac{\partial^{2} \phi}{\partial \xi^{2}}\right) \mathbf{a} \\
\frac{\partial^{2}}{\partial \xi^{2}} \phi & =k_{p}^{2} \gamma_{t}^{2}\left[\beta_{t}\left(1-1 / \psi^{2} \gamma_{t}^{2}\right)^{-1 / 2}-1\right] .
\end{aligned}
$$

Equations (10)-(11) completely describe the nonlinear generation of relativistic harmonic radiation in 1-D within the quasistatic approximation, i.e., $\tau_{L} \ll \tau_{E}$. For the case where the transform velocity, $\beta_{t}$, is set equal to the phase velocity, $\beta_{p h}$, of the incident laser, (10)-(11) may be used to analyze relativistic harmonic generation, as is done in detail in the following. For the case where $\beta_{t}$ is set equal to the group velocity, $\beta_{g}$, of a laser pulse with a pulse length $\simeq \lambda_{p}$, (11) may be used to analyze laser wakefield generation [5]-[9]. The limiting case of $\beta_{t}=1$ has been analyzed in detail in [8].

It is of interest to define the nonlinear index of refraction $n_{R}$ by setting the right side of the wave equation (10) equal to $\left(\omega^{2} / c^{2}\right)\left(1-n_{R}^{2}\right) \mathbf{a}$, which gives

$$
n_{R}^{2}=1-\frac{\omega_{p}^{2}}{\omega^{2}(1+\phi)}\left(1+\frac{1}{k_{p}^{2} \gamma_{t}^{2}} \frac{\partial^{2} \phi}{\partial \xi^{2}}\right)
$$

In particular, the slow part of $n_{R}$ determines the dispersion relation for the radiation field $a$, whereas the fast part of 
$n_{R}$ determines the generation of harmonic radiation, as is discussed below.

\section{A. Dispersion Relation}

To describe the generation of relativistic harmonics, the electrostatic potential $\phi$ is separated into slow and fast components, $\phi=\phi_{s}+\phi_{f}$, where $\phi_{s}$ is approximately constant, $\left|\partial \phi_{s} / \partial \xi\right| \ll\left|k_{0} \phi\right|$, and $\left|\partial \phi_{f} / \partial \xi\right| \sim\left|k_{0} \phi\right|$. The fast component $\phi_{f}$ contains the harmonic content and is important in determining the source current which drives the $N^{\text {th }}$ harmonic radiation. The slow component $\phi_{s}$ is important in determining the dispersive characteristics of the harmonic radiation as well as of the pump laser field. In the following, it is assumed that $\phi_{f}^{2} /\left(1+\phi_{s}\right)^{2} \ll 1$. Furthermore, it is assumed that the vector potential of the harmonic radiation $\mathbf{a}_{N}$, where $N$ is the harmonic number, is much less than that of the pump laser, i.e., $\left|a_{N}\right| /\left|\hat{a}_{0}\right| \ll 1$.

The dispersion relation is determined by examining the slow part of the $n_{R}^{2}$, (12). Assuming $\left|\left(k_{p} \gamma_{p h}\right)^{-2} \partial^{2} \phi_{s} / \partial \xi^{2}\right| \ll 1$, the dispersion relation for the radiation field, $a$, to leading order (i.e., neglecting $\phi_{f}$ ), is given by

$$
\omega_{N}^{2} / c^{2}=k_{N}^{2}+k_{p}^{2} /\left(1+\phi_{s}\right)
$$

where $\mathbf{a}=\hat{\mathbf{a}} \exp \left(i k_{N} z-i \omega_{N} t\right)$ has been assumed with $\hat{\mathbf{a}}$ slowly varying compared to the phase factor $\exp \left(i k_{N} z-\right.$ $\left.i \omega_{N} t\right)$. Equation (13) holds for the pump laser field $\left(\omega_{N}=\right.$ $\left.\omega_{0}, k_{N}=k_{0}\right)$ as well as for the various harmonics. Notice that the transform velocity is the phase velocity of the incident laser field, $\beta_{p h}=\omega_{0} / c k_{0}$, and, hence, $\gamma_{p h}^{-2}=1-\beta_{p h}^{2}=$ $-k_{p}^{2} / k_{0}^{2}\left(1+\phi_{s}\right)<0$. Furthermore, for a nonevolving, long incident laser pulse of the form $a_{0}=\hat{a}_{0} \exp \left(i k_{0} z-i \omega_{0} t\right)$, the quantity $\left(1+\phi_{s}\right)$ is approximately constant, as is discussed in the following.

Consider a long pump laser pulse with a slowly varying envelope, $\left|\partial \hat{a}_{0} / \partial \xi\right| \sim\left|\hat{a}_{0} / c \tau_{L}\right|$, where $\tau_{L}$ is the laser pulse duration, propagating in a plasma with $c \tau_{L} \gg \lambda_{p}, \lambda_{0}$. The slow part of the potential $\phi_{s}$ may be determined from examining the slow part of Poisson's equation (11). In the long pulse limit, $\partial^{2} \phi_{s} / \partial \xi^{2}$ may be neglected and (11) implies that, to leading order (i.e., neglecting $\phi_{f}$ ) [8], [21],

$$
\left(1+\phi_{s}\right)=\left(1+\hat{a}_{0}^{2} / 2\right)^{1 / 2} \text {. }
$$

In particular, notice that in the long pulse limit, $c \tau_{L} \gg \lambda_{p}, \lambda_{0}$, propagation (i.e., real $k$ ) requires $\omega_{p}^{2} / \omega^{2}\left(1+\phi_{s}\right)<1$. This implies that for intense pump laser fields with $\hat{a}_{0} \geq 1$, propagation in an overdense plasma in which $\omega_{p}^{2} / \omega^{2} \geq 1$ may be possible. Physically, $\gamma_{s}=1+\phi_{s}=\left(1+\hat{a}_{0}^{2} / 2\right)^{1 / 2}$, and the reduction in the effective plasma frequency, $\omega_{p} / \gamma_{s}^{1 / 2}$, is due to the relativistic quiver motion of the electrons.

It should be emphasized that (14) only applies to the long pulse limit $c \tau_{L} \gg \lambda_{p}, \lambda_{0}$. For $\tau_{L}=1 \mathrm{psec}, \tau_{L} \gg \lambda_{p}$ implies plasma densities $n_{0} \gg 10^{16} \mathrm{~cm}^{-3}$. In the short pulse limit, $\lambda_{p} \gg c \tau_{L} \gg \lambda_{0}$, it can be shown [8], [21] that $\left|\phi_{s}\right| \ll 1$ provided $c \tau_{L} \ll \lambda_{p} /\left(1+\hat{a}_{0}^{2} / 2\right)^{1 / 2}$. Physically, $\left|\phi_{s}\right| \ll 1$ holds for pulses sufficiently shorter than $\lambda_{p}$, since $\lambda_{p}$ is the characteristic length scale (in the $\xi$ frame) for collective electron motion (i.e., collective electron motion leading to charge separation and significant values of $\phi_{s}$ does not occur on time scales sufficiently shorter than $1 / \omega_{p}$ ).

\section{B. Plasma Response}

The source current driving the harmonic radiation is determined by the harmonic content of $n_{R}$. As indicated by (12), $n_{R}$ may be specified entirely in terms of $\phi$. Hence, it is necessary to determine the various harmonic components of $\phi$. This is done by analyzing the plasma response to the pump laser field, $a_{0}=\hat{a}_{0} \cos k_{0} \xi$, via Poisson's equation (11). The various harmonic components of $\phi_{f}=\sum_{\ell} \phi_{2 \ell}$, where $\phi_{2 \ell} \sim \cos \left(2 \ell k_{0} \xi\right)$ with $\ell=1,2,3 \ldots$, may be determined analytically in the limit $\epsilon=k_{p}^{2} / k_{0}^{2}\left(1+\phi_{s}\right)<1$. In this limit $\left|\phi_{2 \ell} /\left(1+\phi_{s}\right)\right| \sim \epsilon^{\ell}$, and the various harmonic components of $\phi_{f}$ may be solved order by order in $\epsilon$.

To solve for $\phi_{f}$ via (11), two expansions will be used. Assuming $\left|\left(\psi \gamma_{p h}\right)^{-2}\right| \leq \epsilon$, gives

$$
\left(1-\frac{1}{\psi^{2} \gamma_{p h}^{2}}\right)^{-1 / 2}=\sum_{\ell=0}^{\infty} \frac{(2 \ell-1) ! !}{(2 \ell) ! !}\left(\frac{1}{\psi^{2} \gamma_{p h}^{2}}\right)^{\ell},
$$

where $(2 \ell-1) ! !=1 \cdot 3 \cdot \ldots \cdot(2 \ell-1)$ and $(2 \ell) ! !=2 \cdot 4 \cdot \ldots \cdot(2 \ell)$. Assuming $\left|\phi_{f} /\left(1+\phi_{s}\right)\right| \leq \epsilon$, gives

$$
\begin{aligned}
\left(\frac{1}{\psi^{2} \gamma_{p h}^{2}}\right)^{\ell}= & {\left[\frac{\left(1+a^{2}\right)}{\gamma_{p h}^{2}\left(1+\phi_{s}\right)^{2}}\right]^{\ell} } \\
& \cdot \sum_{m=0}^{\infty} \frac{2 \ell(2 \ell+1) \ldots(2 \ell+m-1)}{m !} \\
& \cdot\left[\frac{-\phi_{f}}{\left(1+\phi_{s}\right)}\right]^{m} .
\end{aligned}
$$

Letting $\phi_{f}=\sum_{\ell} \phi_{2 \ell}$, where $\phi_{2 \ell} \sim \epsilon^{\ell} \cos \left(2 \ell k_{0} \xi\right)$ for $\ell=$ $1,2,3 \ldots$, and using the expansions in (15a) and (15b), allows $\phi_{2 \ell}$ to be solved order by order in $\epsilon$. For example, $\phi_{2}$ (order $\epsilon), \phi_{4}\left(\right.$ order $\left.\epsilon^{2}\right)$ and $\phi_{6}\left(\right.$ order $\epsilon^{3}$ ) are given by

$$
\begin{gathered}
\frac{\partial^{2}}{\partial \xi^{2}} \phi_{2}=\frac{k_{p}^{2}\left(a_{0}^{2}\right)_{2}}{2\left(1+\phi_{s}\right)^{2}}, \\
\frac{\partial^{2}}{\partial \xi^{2}} \phi_{4}=k_{p}^{2}\left[-\frac{\phi_{2}\left(a_{0}^{2}\right)_{2}}{\left(1+\phi_{s}\right)^{3}}+\frac{3\left(a_{0}^{4}\right)_{4}}{8 \gamma_{p h}^{2}\left(1+\phi_{s}\right)^{4}}\right], \\
\frac{\partial^{2}}{\partial \xi^{2}} \phi_{6}=\frac{-k_{p}^{2}\left(a_{0}^{2}\right)_{2}}{\left(1+\phi_{s}\right)^{3}}\left[\phi_{4}-\frac{3 \phi_{2}^{2}}{2\left(1+\phi_{s}\right)}\right. \\
\left.+\frac{3\left(a_{0}^{2}\right)_{2}}{2 \gamma_{p h}^{2}\left(1+\phi_{s}\right)^{2}}\left(\phi_{2}-\frac{5\left(a_{0}^{2}\right)_{2}}{24 \gamma_{p h}^{2}\left(1+\phi_{s}\right)^{2}}\right)\right](16 \mathrm{c})
\end{gathered}
$$

where the subscript $(Q)_{\ell}$ refers to the $\ell^{\text {th }}$ harmonic component of $Q$, i.e., $(Q)_{\ell} \sim \exp \left(\ell k_{0} \xi\right)$. These equations can be solved iteratively to yield

$$
\begin{aligned}
& \phi_{2}=-\frac{k_{p}^{2} \hat{a}_{0}^{2} \cos 2 k_{0} \xi}{16 k_{0}^{2}\left(1+\phi_{s}\right)^{2}}, \\
& \phi_{4}=\frac{k_{p}^{4} \hat{a}_{0}^{4} \cos 4 k_{0} \xi}{2^{9} k_{0}^{4}\left(1+\phi_{s}\right)^{5}},
\end{aligned}
$$




$$
\phi_{6}=-\frac{17 k_{p}^{6} \hat{a}_{0}^{6} \cos 6 k_{0} \xi}{9 \cdot 2^{14} k_{0}^{6}\left(1+\phi_{s}\right)^{8}}
$$

For order of magnitude scaling purposes, (17a)-(17c) imply that the $2 \ell^{\text {th }}$ harmonic component of $\phi_{f}$ scales as

$$
\phi_{2 \ell} \sim(-1)^{\ell} \frac{(2 \ell-1) ! !}{(2 \ell) ! !}\left(\frac{k_{p}}{2 k_{0}}\right)^{2 \ell} \frac{\hat{a}_{0}^{2 \ell} \cos 2 \ell k_{0} \xi}{2 \ell^{2}\left(1+\phi_{s}\right)^{3 \ell-1}} .
$$

Knowing the harmonic components of $\phi_{f}$, it is possible to determine the harmonic components of the source current $S$ which drives the harmonic radiation. The source current $S$ is given by the right side of (10),

$$
S=\frac{k_{p}^{2} a}{(1+\phi)}\left(1+\frac{1}{k_{p}^{2} \gamma_{p h}^{2}} \frac{\partial^{2} \phi}{\partial \xi^{2}}\right)
$$

Using the expansion

$$
(1+\phi)^{-1}=\left(1+\phi_{s}\right)^{-1} \sum_{m=0}^{\infty}\left[-\phi_{f}\left(1+\phi_{s}\right)^{-1}\right]^{m}
$$

allows the harmonic components of $S$ to be solved order by order in $\epsilon$. For example, the third, $S_{3}$, fifth, $S_{5}$, and seventh, $S_{7}$, harmonic components are given by (21) at the bottom of this page. Using (17a)-(17c) gives

$$
\begin{aligned}
S_{3} & =-\frac{3 k_{p}^{4} \hat{a}_{0}^{3} \cos 3 k_{0} \xi}{32 k_{0}^{2}\left(1+\phi_{s}\right)^{4}}, \\
S_{5} & =\frac{3 k_{p}^{6} \hat{a}_{0}^{5} \cos 5 k_{0} \xi}{2^{8} k_{0}^{4}\left(1+\phi_{s}\right)^{7}} \\
S_{7} & =-\frac{5 k_{p}^{8} \hat{a}_{0}^{7} \cos 7 k_{0} \xi}{3 \cdot 2^{10} k_{0}^{6}\left(1+\phi_{s}\right)^{10}} .
\end{aligned}
$$

For order of magnitude scaling purposes, (22a)-(22c) imply that the $(2 \ell+1)^{\text {th }}$ harmonic component of $S$ scales as

$$
S_{2 \ell+1} \sim(-1)^{\ell} k_{p}^{2} \frac{(2 \ell-1) ! !}{(2 \ell) ! !}\left(\frac{k_{p}}{2 k_{0}}\right)^{2 \ell} \frac{\hat{a}_{0}^{2 \ell+1} \cos (2 \ell+1) k_{0} \xi}{\ell\left(1+\phi_{s}\right)^{3 \ell+1}}
$$

\section{Harmonic Radiation}

The above expressions for the harmonic components of the source current, $S_{2 \ell+1}$, may be used in the wave equation (10), to determine the growth and saturation of the relativistic harmonic radiation. It is convenient to represent the $N^{\text {th }}$ harmonic radiation field, $a_{N}$, by the form

$$
a_{N}=\hat{a}_{N}(\tau) \exp \left(i k_{N} \xi-i \Delta \omega_{N} \tau\right)
$$

with $\Delta \omega_{N}=\omega_{N}-\beta_{p h} c k_{N}$ and $k_{N} \xi-\Delta \omega_{N} \tau=k_{N} z-\omega_{N} t$, where $k_{N}$ and $\omega_{N}$ are the wavenumber and frequency of the $N^{t h}$ harmonic radiation field. Inserting this into (10) gives a reduced wave equation for the harmonic amplitude $\hat{a}_{N}$

$$
\left(\frac{\partial}{\partial \tau}-2 i \omega_{N}\right) \frac{\partial}{\partial \tau} \hat{a}_{N}=-c^{2} \hat{S}_{N} \exp \left(i \Delta \omega_{N} \tau\right)
$$

where $\omega_{N}$ and $k_{N}$ satisfy the dispersion relation, (13), with $k_{N}=N k_{0}$ and $N=2 \ell+1$. The amplitude of the source current for the $N^{\text {th }}$ harmonic, $\hat{S}_{N}$, is given by $S_{N}=$ $\hat{S}_{N} \exp \left(i N k_{0} \xi\right)$, where $S_{N}$ is given in $(22)-(23)$.

The reduced wave equation (25) has the solution

$$
\hat{a}_{N} \simeq-c^{2} \hat{S}_{N} \frac{\left[\exp \left(i \Delta \omega_{N} \tau\right)-1\right]}{\left(\omega_{N}^{2}-c^{2} \beta_{p h}^{2} k_{N}^{2}\right)}
$$

where

$$
\omega_{N}^{2}-c^{2} \beta_{p h}^{2} k_{N}^{2}=-\frac{c^{2} k_{p}^{2}}{\left(1+\phi_{s}\right)}\left(N^{2}-1\right)
$$

For small times $\left|\Delta \omega_{N} \tau\right| \ll 1$, the harmonics grow linearly in time [8],

$$
\hat{a}_{N} \simeq-i \hat{S}_{N} \frac{c \tau}{2 N k_{0}}
$$

The $N^{\text {th }}$ harmonic reaches its maximum amplitude [1], [19], [20] after a dephasing length $c \tau_{d_{N}}=\pi c /\left|\Delta \omega_{N}\right|$, where $\Delta \omega_{N}=c k_{N}\left(\beta_{N}-\beta_{p h}\right)$ and $\beta_{N}=\omega_{N} / c k_{N}$, i.e.,

$$
\begin{aligned}
\Delta \omega_{N}=N c k_{0} & {\left[\left(1+\frac{k_{p}^{2}}{N^{2} k_{0}^{2}\left(1+\phi_{s}\right)}\right)^{1 / 2}\right.} \\
& \left.-\left(1+\frac{k_{p}^{2}}{k_{0}^{2}\left(1+\phi_{s}\right)}\right)^{1 / 2}\right] .
\end{aligned}
$$

For $k_{p}^{2} / k_{0}^{2}\left(1+\phi_{s}\right) \ll 1,(29)$ may be expanded giving

$$
c \tau_{d_{N}} \simeq \frac{N \lambda_{p}^{2}\left(1+\phi_{s}\right)}{\left(N^{2}-1\right) \lambda_{0}}
$$

$$
\begin{aligned}
S_{3}=\frac{k_{p}^{2} a}{\left(1+\phi_{s}\right)} & {\left[-\frac{\phi_{2}}{\left(1+\phi_{s}\right)}+\frac{1}{k_{p}^{2} \gamma_{p h}^{2}} \frac{\partial^{2} \phi_{2}}{\partial \xi^{2}}\right], } \\
S_{5}=\frac{-k_{p}^{2} a}{\left(1+\phi_{s}\right)} & {\left[\frac{\phi_{4}}{\left(1+\phi_{s}\right)}-\frac{\phi_{2}^{2}}{\left(1+\phi_{s}\right)^{2}}-\frac{1}{k_{p}^{2} \gamma_{p h}^{2}}\left(\frac{\partial^{2} \phi_{4}}{\partial \xi^{2}}-\frac{\phi_{2}}{\left(1+\phi_{s}\right)} \frac{\partial^{2} \phi_{2}}{\partial \xi^{2}}\right)\right], } \\
S_{7}=\frac{-k_{p}^{2} a}{\left(1+\phi_{s}\right)} & \left\{\frac{\phi_{6}}{\left(1+\phi_{s}\right)}+\frac{\phi_{2}^{3}}{\left(1+\phi_{s}\right)^{3}}-\frac{2 \phi_{2} \phi_{4}}{\left(1+\phi_{s}\right)^{2}}\right. \\
& \left.-\frac{1}{k_{p}^{2} \gamma_{p h}^{2}}\left[\frac{\partial^{2} \phi_{6}}{\partial \xi^{2}}-\frac{\phi_{2}}{\left(1+\phi_{s}\right)} \frac{\partial^{2} \phi_{4}}{\partial \xi^{2}}-\left(\frac{\phi_{4}}{\left(1+\phi_{s}\right)}-\frac{\phi_{2}^{2}}{\left(1+\phi_{s}\right)^{2}}\right) \frac{\partial^{2} \phi_{2}}{\partial \xi^{2}}\right]\right\} .
\end{aligned}
$$




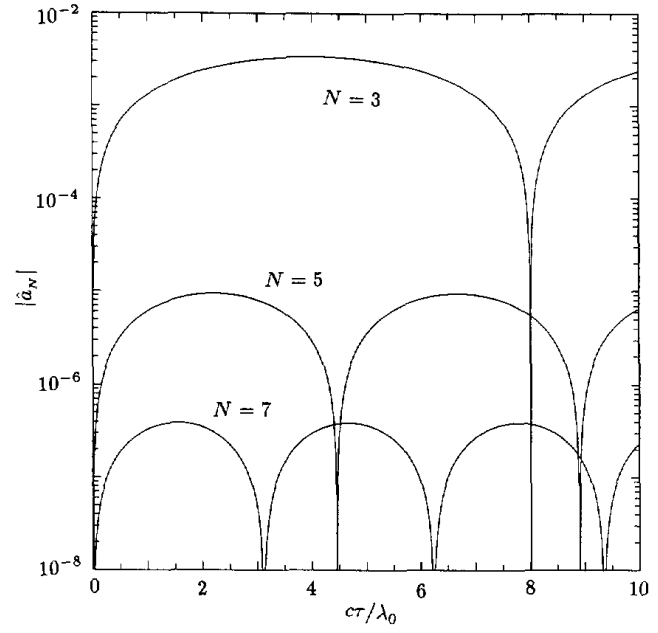

Fig. 2. The amplitude of the normalized vector potential of the harmonic radiation, $\left|\hat{a}_{N}\right|$, for the first three harmonics, $N=3,5$, and 7 , versus the normalized propagation distance, $c \tau / \lambda_{0}$, for a $\lambda_{0}=1 \mu \mathrm{m}$ wavelength lase of intensity $I_{0}=6.7 \times 10^{18} \mathrm{~W} / \mathrm{cm}^{2}\left(\hat{a}_{0}=2.2\right)$ interacting with a plasma of density $n_{0}=10^{20} \mathrm{~cm}^{-3}\left(\lambda_{p}=3.4 \mu \mathrm{m}\right)$.

The maximum amplitude of the $N^{t h}$ harmonic at $c \tau=c \tau_{d_{N}}$ is given by

$$
\left|\hat{a}_{N}\right|_{\text {max }}=\frac{2\left|\hat{S}_{N}\right|\left(1+\phi_{s}\right)}{k_{p}^{2}\left(N^{2}-1\right)} .
$$

In particular, for the third, fifth, and seventh harmonics,

$$
\begin{aligned}
\left|\hat{a}_{3}\right|_{\text {max }} & =\frac{3 k_{p}^{2} \hat{a}_{0}^{3}}{2^{7} k_{0}^{2}\left(1+\phi_{s}\right)^{3}}, \\
\left|\hat{a}_{5}\right|_{\text {max }} & =\frac{k_{p}^{4} \hat{a}_{0}^{5}}{2^{10} k_{0}^{4}\left(1+\phi_{s}\right)^{6}}, \\
\left|\hat{a}_{7}\right|_{\text {max }} & =\frac{5 k_{p}^{6} \hat{a}_{0}^{7}}{9 \cdot 2^{13} k_{0}^{6}\left(1+\phi_{s}\right)^{9}} .
\end{aligned}
$$

An order of magnitude expression for the maximum amplitude of the $(2 \ell+1)$ harmonic is given by

$$
\left|\hat{a}_{2 \ell+1}\right|_{\max } \sim \frac{2(2 \ell-1) ! !\left(k_{p} / 2 k_{0}\right)^{2 \ell} \hat{a}_{0}^{2 \ell+1}}{\ell(2 \ell) ! !\left[(2 \ell+1)^{2}-1\right]\left(1+\phi_{2}\right)^{3 \ell}} .
$$

Equations (32)-(33) are valid for all $\hat{a}_{0}$, including $\hat{a}_{0} \geq 1$. It is easily verified that $\left|\hat{a}_{N}\right|_{\max } \ll \hat{a}_{0}$. A plot of the harmonic radiation amplitude $\left|\hat{a}_{N}\right|$ verses the normalized propagation distance $c \tau / \lambda_{0}$ is shown in Fig. 2 for the first three harmonics for the parameters $\lambda_{0}=1 \mu \mathrm{m}, \lambda_{p}=3.4 \mu \mathrm{m}\left(n_{0}=10^{20}\right.$ $\left.\mathrm{cm}^{-3}\right)$ and $\hat{a}_{0}=2.2\left(I_{0}=6.7 \times 10^{18} \mathrm{~W} / \mathrm{cm}^{2}\right)$.

At saturation for the $N^{\text {th }}$ harmonic, i.e., $c \tau=c \tau_{d_{N}}$, the ratio of the power in the $N^{\text {th }}$ harmonic, $P_{N}$, to the power in the pump radiation field, $P_{0}$, is given by $P_{N} / P_{0}=N^{2}\left|\hat{a}_{N}\right|^{2} / \hat{a}_{0}^{2}$. Using (32)-(33), this can be written as

$$
\frac{P_{N}}{P_{0}} \simeq C_{N}\left(\frac{\lambda_{0} \hat{a}_{0}}{\lambda_{p}}\right)^{2(N-1)}\left(1+\frac{\hat{a}_{0}^{2}}{2}\right)^{-3(N-1) / 2}
$$

where $C_{N}$ are constants which decrease rapidly with increasing harmonic number, i.e., $C_{3}=4.9 \times 10^{-3}, C_{5}=2.4 \times$
$10^{-5}$ and $C_{7}=2.3 \times 10^{-7}$. Furthermore, driven relativistic harmonic generation is a nonresonant interaction; hence, the process is not sensitive to thermal plasma effects.

As an example, consider an incident laser with $P_{0}=10 \mathrm{TW}$, $\lambda_{0}=1 \mu \mathrm{m}$ and spot size $r_{0}=10 \mu \mathrm{m}\left(\hat{a}_{0}=2.2\right)$, interacting with a plasma of density $n_{0}=10^{20} \mathrm{~cm}^{-3}\left(\lambda_{p}=3.4 \mu \mathrm{m}\right)$. For the third (and fifth) harmonic, $P_{N} / P_{0}=2.2 \times 10^{-5}$ $\left(4.6 \times 10^{-10}\right)$ and $L_{d}=8.0 \mu \mathrm{m}(4.3 \mu \mathrm{m})$. Hence, $220 \mathrm{MW}$ $(4.6 \mathrm{~kW})$ should be observed at a wavelength of $\lambda=3300 \AA$ $(2000 \AA)$. Clearly, the limitations due to phase detuning are restrictive. If a scheme for phase matching could be conceived, the interaction distance, $L$, and thus the harmonic power, $P_{N} \sim L^{2}$, could be increased.

\section{DIFFRACTIVE EFFECTS}

The above nonlinear theory indicates that the $N^{\text {th }}$ harmonic saturates (i.e., reaches maximum amplitude) after a detuning distance $c \tau_{d_{N}}=\pi c /\left|\Delta \omega_{N}\right|$, where $\Delta \omega_{N}=c k_{N}\left(\beta_{N}-\beta_{p h}\right)$. In particular, for the third harmonic, $c \tau_{d_{3}} \simeq 3 \lambda_{p}^{2}\left(1+\phi_{s}\right) / 8 \lambda_{0}$. Physically, saturation by detuning arises due to the fact that the phase velocity of the fundamental pump radiation is greater that than the phase velocity of the harmonic radiation, i.e., $\beta_{p h}>\beta_{N}$. In a realistic 3-D configuration, however, diffractive effects will limit the effective radiation-plasma interaction length to a few vacuum Rayleigh lengths, $Z_{R}$, where $Z_{R}=k_{0} r_{0}^{2} / 2$ and $r_{0}$ is the minimum spot size of the radiation field (assumed to be Gaussian). For interaction distances $L \ll \pi Z_{R}$, diffraction effects are unimportant and the 1-D theory is an adequate description. In the limit $\pi Z_{R} \lesssim L$, however, and in particular $\pi Z_{R} \lesssim c \tau_{d_{N}}$, diffractive effects are important and must be included in the analysis.

In the following, the generation of second and third harmonic radiation is analyzed using 3-D relativistic fluid equations in the limit $a_{0}^{2} \ll 1$. Higher order harmonic generation can be consider by solving the fluid equations to higher order in $a_{0}^{2}$. Furthermore, the radiation fields are assumed to undergo vacuum diffraction. Notice that in the regime in which 3-D effects are important, the condition $\pi Z_{R}<$ $c \tau_{d_{3}}$ implies $\delta=2 k_{p}^{2} r_{0}^{2} / 3<1$. Hence, the effects of relativistic optical guiding [3]-[10], which become important when $P / P_{c}=k_{p}^{2} r_{0}^{2} \hat{a}_{0}^{2} / 32 \gtrsim 1$, may be neglected in the 3-D regime when $\hat{a}_{0}^{2} \ll 1$. Assuming vacuum diffraction, a long pulse, Gaussian, incident radiation field evolves according to $a(r, z, t)=a_{v} \exp i \theta_{0}$, where $\theta_{0}=k_{0} z-\omega_{0} t$ and the radiation envelope $a_{v}$ is given by [24]

$$
a_{v}(r, z)=\frac{\hat{a}_{0} r_{0}}{r_{s}} \exp \left[-(1-i \alpha) \frac{r^{2}}{r_{s}^{2}}-i \tan ^{-1} \alpha\right]
$$

where $r_{s}=r_{0}\left(1+\alpha^{2}\right)^{1 / 2}$ is the radiation spot size, $\alpha=z / Z_{R}$ and $z=0$ is the location of the laser focus at which $r_{s}=r_{0}$.

\section{A. 3-D Formulation}

The 3-D pump laser interaction with the plasma electrons will be modeled using the cold, relativistic fluid equations. In particular, the momentum equation and the continuity equation may be written as

$$
\frac{1}{c} \frac{d}{d t} \mathbf{u}=\nabla \phi+\frac{1}{c} \frac{\partial}{\partial t} \mathbf{a}-\frac{1}{\gamma} \mathbf{u} \times(\nabla \times \mathbf{a})
$$




$$
\frac{1}{c} \frac{\partial}{\partial t}(\rho \gamma)+\nabla \cdot(\rho \mathbf{u})=0
$$

respectively, where $\mathbf{u}=\mathbf{p} / m c$ is the normalized momentum, $\gamma=\left(1+u^{2}\right)^{1 / 2}$ is the relativistic factor, and $\rho=n /\left(n_{0} \gamma\right)$. The evolution of the normalized potentials are determined from the wave equation and Poisson's equation, which may be written as

$$
\begin{gathered}
\left(\nabla^{2}-\frac{1}{c^{2}} \frac{\partial^{2}}{\partial t^{2}}\right) \mathbf{a}=k_{p}^{2} \rho \mathbf{u}+\frac{1}{c} \frac{\partial}{\partial t} \nabla \phi \\
\nabla^{2} \phi=k_{p}^{2}(\rho \gamma-1)
\end{gathered}
$$

respectively, where Coulomb gauge, $\nabla \cdot \mathbf{a}=0$, has been assumed.

To study the generation of the third harmonic, the above equations will be solved perturbatively, i.e., order by order in the pump laser amplitude $\hat{a}_{0}$, assuming $\hat{a}_{0}^{2} \ll 1$. The various plasma quantities, denoted by $Q$, will be represented by an expansion $Q=Q_{0}+Q_{1}+Q_{2}+\ldots$, where $Q_{n} \sim a_{0}^{n}$. The pump laser vector potential is assumed to be known and given by $\mathbf{a}_{0 \perp}=a_{v} \exp i \theta_{0} \mathbf{e}_{x}$, where $a_{v}(r, z)$ is the vacuum solution given by (35). The effects of pump depletion and of various laser-plasma instabilities will be neglected. The zeroth order plasma quantities (in the absence of the pump laser) are given by $\mathbf{u}_{0}=0, \gamma_{0}=1, \rho_{0}=1$ and $\phi_{0}=0$, i.e., the plasma is assumed to be initially uniform, stationary, and neutral. To first order in the normalized vector potential, (36) implies that $\mathbf{u}_{1}=\mathbf{a}_{0}$, which is simply the quiver motion of the electrons. Furthermore, (36)-(39) imply $\rho_{1}=\gamma_{1}=\phi_{1}=0$. The first order form of the wave equation (38) implies that the pump laser field obeys the dispersion relation $\omega_{0}^{2} / c^{2}=k_{0}^{2}+k_{p}^{2}$.

To second order, (36)-(39) imply that quantities $\phi_{2}, \mathbf{u}_{2}$ and $\rho_{2}$ are related to $a_{0}^{2}$ by

$$
\begin{aligned}
& \left(\frac{1}{c^{2}} \frac{\partial^{2}}{\partial t^{2}}+k_{p}^{2}\right) \phi_{2}=k_{p}^{2} \frac{a_{0}^{2}}{2} \\
& \left(\frac{1}{c^{2}} \frac{\partial^{2}}{\partial t^{2}}+k_{p}^{2}\right) \mathbf{u}_{2}=-\frac{1}{c} \frac{\partial}{\partial t} \nabla \frac{a_{0}^{2}}{2}, \\
& \left(\frac{1}{c^{2}} \frac{\partial^{2}}{\partial t^{2}}+k_{p}^{2}\right) \rho_{2}=\left(\nabla^{2}-\frac{1}{c^{2}} \frac{\partial^{2}}{\partial t^{2}}-k_{p}^{2}\right) \frac{a_{0}^{2}}{2} .
\end{aligned}
$$

Furthermore, (40a) and (40c) imply that the second-order wave equation describing the generation of the second harmonic may be written as

$$
\left(\nabla^{2}-\frac{1}{c^{2}} \frac{\partial^{2}}{\partial t^{2}}-k_{p}^{2}\right) \mathbf{a}_{2}=0
$$

Hence, no second harmonic radiation will be generated.

To third order, (36)-(39) imply that quantities $\phi_{3}$ and $\mathbf{u}_{3}$ are related to $a_{0}^{3}$ by

$$
\begin{aligned}
\left(\frac{1}{c^{2}} \frac{\partial^{2}}{\partial t^{2}}+k_{p}^{2}\right) \nabla^{2} \phi_{3} & =k_{p}^{2}\left[\nabla^{2}\left(\mathbf{a}_{0} \cdot \mathbf{u}_{2}\right)-\frac{1}{c} \frac{\partial}{\partial t} \nabla \cdot\left(\mathbf{a}_{0} \rho_{2}\right)\right], \\
\frac{1}{c} \frac{\partial}{\partial t} \mathbf{u}_{3} & =\nabla \phi_{3}-\nabla\left(\mathbf{a}_{0} \cdot \mathbf{u}_{2}\right)
\end{aligned}
$$

Furthermore, the third-order transverse wave equation, describing the generation of third harmonic radiation, is given by

$$
\begin{aligned}
& \left(\nabla^{2}-\frac{1}{c^{2}} \frac{\partial^{2}}{\partial t^{2}}-k_{p}^{2}\right) \mathbf{a}_{3 \perp} \\
& \quad=\left(k_{p}^{2} \rho_{2} \mathbf{a}_{0 \perp}+k_{p}^{2} \mathbf{u}_{3 \perp}+\frac{1}{c} \frac{\partial}{\partial t} \nabla_{\perp} \phi_{3}\right)_{3} .
\end{aligned}
$$

where $(\ldots)_{n}$ signifies the $n^{\text {th }}$ harmonic component.

In particular, to determine the source term driving the third harmonic radiation, it is necessary to determine the fast (i.e., third harmonic) components of $\phi_{3}$ and $\mathbf{u}_{3}$. Assuming $\phi_{3}, \mathbf{u}_{3} \sim \exp 3 i \theta_{0},(42 a)$ and $(42 b)$ imply

$$
\begin{aligned}
& \phi_{3} \simeq-\frac{\omega_{p}^{2}}{9 \omega_{0}^{2}}\left(\mathbf{a}_{0} \cdot \mathbf{u}_{2}\right)_{3}, \\
& \mathbf{u}_{3} \simeq-\frac{i c}{3 \omega_{0}} \nabla\left(\mathbf{a}_{0} \cdot \mathbf{u}_{2}\right)_{3}
\end{aligned}
$$

where terms of order $k_{p}^{2} / k_{0}^{2} \ll 1$ and $1 / r_{0}^{2} k_{0}^{2} \ll 1$ have been neglected. It can be shown that the leading order contributions of each of the three terms on the right side of (43) are of the same order. However, to leading order, $k_{p}^{2} \mathbf{u}_{3 \perp}+$ $\nabla_{\perp} \partial \phi_{3} / \partial c t=0$, and, hence, the wave equation describing third harmonic generation is given by

$$
\left(\nabla^{2}-\frac{1}{c^{2}} \frac{\partial^{2}}{\partial t^{2}}-k_{p}^{2}\right) \mathbf{a}_{3 \perp}=k_{p}^{2}\left(\rho_{2} \mathbf{a}_{0 \perp}\right)_{3}
$$

where higher order terms (of order $k_{p}^{2} / k_{0}^{2}$ and $1 / r_{0}^{2} k_{0}^{2}$ ) have been neglected. The transverse wave equation (45) along with the second harmonic component of $\rho_{2}$,

$$
\left(\frac{1}{c^{2}} \frac{\partial^{2}}{\partial t^{2}}+k_{p}^{2}\right) \rho_{2}=\left(\nabla^{2}-\frac{1}{c^{2}} \frac{\partial^{2}}{\partial t^{2}}-k_{p}^{2}\right) \frac{\left(a_{0}^{2}\right)_{2}}{2}
$$

completely determine the generation of third harmonic radiation in 3-D in the limit $a_{0}^{2} \ll 1$.

\section{B. Third Harmonic Generation}

The pump laser is assumed to be a long pulse, Gaussian laser beam which is diffracting according to the relation $a_{0}(r, z, t)=a_{v} \exp i \theta_{0}$, where $a_{v}(r, z)$ is the envelope given by (35) and $\omega_{0}^{2} / c^{2}=k_{p}^{2}+k_{0}^{2}$. Using this form in (46) gives

$$
\rho_{2} \simeq-\frac{1}{2 r_{0}^{2} k_{0}^{2}}\left[\frac{3}{8} r_{0}^{2} k_{p}^{2}+\frac{1}{(1+i \alpha)}\right] a_{v}^{2} \exp 2 i \theta_{0} .
$$

Hence, the source term driving the third harmonic radiation is given by

$$
k_{p}^{2}\left(\rho_{2} a_{0}\right)_{3} \simeq-\frac{k_{p}^{2}}{4 r_{0}^{2} k_{0}^{2}}\left[\frac{3}{8} r_{0}^{2} k_{p}^{2}+\frac{1}{(1+i \alpha)}\right] a_{v}^{3} \exp 3 i \theta_{0} .
$$

It is convenient to denote the third harmonic radiation field, $a_{3}(r, z, t)$, by the following form,

$$
\begin{aligned}
a_{3}(r, z, t) & =f(z) a_{v 3}(r, z) \exp i \theta_{3}, \\
a_{v 3}(z, r) & =\frac{r_{3}}{r_{s 3}} \exp \left[-\left(1-i \alpha_{3}\right) \frac{r^{2}}{r_{s 3}^{2}}-i \tan ^{-1} \alpha_{3}\right]
\end{aligned}
$$


where $\theta_{3}=k_{3} z-\omega_{3} t, \omega_{3}$ and $k_{3}$ are the third harmonic frequency and wavenumber, $r_{s 3}=r_{3}\left(1+\alpha_{3}^{2}\right)^{1 / 2}$ is the third harmonic spot size, $\alpha_{3}=z / Z_{R 3}, Z_{R 3}=k_{3} r_{3} / 2$ is the Rayleigh length associated with the third harmonic, and $\omega_{3}^{2} / c^{2}=k_{3}^{2}+k_{p}^{2}$. Inserting this into the wave equation (45) gives

$$
2 i k_{3} a_{v 3} \exp \left(i \theta_{3}\right) \frac{\partial}{\partial z} f \simeq k_{p}^{2}\left(\rho_{2} a_{0}\right)_{3}
$$

where the right side is given by (48). Letting $\omega_{3}=3 \omega_{0}$ and $r_{3}^{2}=r_{0}^{2} / 3$ implies $Z_{R 3} \simeq Z_{R}$ and

$$
\frac{\partial}{\partial z} f \simeq \frac{i k_{p}^{2} \hat{a}_{0}^{3}}{24 r_{0}^{2} k_{0}^{3}}\left[\frac{3}{8} r_{0}^{2} k_{p}^{2}+\frac{1}{(1+i \alpha)}\right] \frac{\exp (i \Delta k z)}{(1+i \alpha)^{2}}
$$

where $\Delta k=3 k_{0}-k_{3}=-4 k_{p}^{2} / 3 k_{0}$.

For a pump laser pulse which interacts with a uniform plasma extending from $z_{\min }<z<z_{\max }$, (51) can be integrated giving

$$
\begin{aligned}
f=\left|f_{1-D}\right| & {\left[h\left(\delta, \alpha_{\max }\right)-h\left(\delta, \alpha_{\min }\right)\right], } \\
h(\delta, \alpha)=-\frac{4}{9}\{ & \left(\frac{\delta}{8}+\frac{1}{(1+i \alpha)}\right) \frac{e^{-i \delta \alpha}}{(1+i \alpha)} \\
& \left.-\frac{\delta^{2}}{8} e^{\delta} E_{1}[\delta(1+i \alpha)]\right\}
\end{aligned}
$$

where $\left|f_{1-D}\right|=\left|3 \hat{a}_{0}^{3} k_{p}^{2} / 2^{7} k_{0}^{2}\right|$ is the maximum amplitude of the third harmonic in the 1-D limit, $\delta=|\Delta k| Z_{R}=2 k_{p}^{2} r_{0}^{2} / 3$ $\left(\delta=\pi Z_{R} / c \tau_{d_{3}}\right), \alpha_{\min }=z_{\min } / Z_{R}, \alpha_{\max }=z_{\max } / Z_{R}$ and $E_{1}$ is the exponential integral.

In particular, consider a finite slab plasma centered about the focal point of the pump laser (chosen to be $z=0$ ) which extends from $z_{\min }=-z_{0}$ to $z_{\max }=z_{0}$. The amplitude function $f$ of the third harmonic signal emerging from the slab plasma at $z=z_{0}$ is given by

$$
f=2\left|f_{1-D}\right| \operatorname{Im}\left[h\left(\delta, \alpha_{0}\right)\right]
$$

where $\operatorname{Im}\left[h\left(\delta, \alpha_{0}\right)\right]$ is the imaginary part of $h\left(\delta, \alpha_{0}\right)$ and $\alpha_{0}=z_{0} / Z_{R}$. The ratio of the third harmonic power to the pump laser power is given by

$$
P_{3} / P_{0}=\omega_{3}^{2} r_{3}^{2}|f|^{2} / \omega_{0}^{2} r_{0}^{2} \hat{a}_{0}^{2}=3|f|^{2} / \hat{a}_{0}^{2} .
$$

Hence, for a slab plasma centered about the laser focus, $P_{3} \sim 4\left|\operatorname{Im}\left[h\left(\delta, \alpha_{0}\right)\right]\right|^{2}$. A plot of the function $H\left(\delta, \alpha_{0}\right)=$ $4\left|\operatorname{Im}\left[h\left(\delta, \alpha_{0}\right)\right]\right|^{2}$ verses the normalized plasma slab width, $\alpha_{0}$, is shown in Fig. 3 for $\delta=0.1,1.0$, and 10.0. Notice that for $\delta=10.0\left(c \tau_{d_{3}} \ll \pi Z_{R}\right)$ and $\alpha_{0} \ll 1$, the first peak, which occurs when the slab width is equal to the detuning length, $2 z_{0}=c \tau_{d_{3}}$, is close to the 1-D value of $H\left(\delta, \alpha_{0}\right)=1$. For $\delta=0.1\left(c \tau_{d_{3}} \gg \pi Z_{R}\right)$, the maximum amplitude of the third harmonic occurs for a plasma length of $\simeq Z_{R}$, at which $H\left(\delta, \alpha_{0}\right) \simeq 1 / 3$. In the limit of a slab plasma with a spatial extent large compared to $Z_{R}$, i.e., $z_{0} \gg Z_{R}\left(\alpha_{0} \gg 1\right)$, $f \rightarrow f_{I}$, where $f_{I}=\left|f_{1-D}\right| \alpha_{0}^{-2} \sin \left(\delta \alpha_{0}\right)$. Hence, $f_{I} \rightarrow 0$ as $\alpha_{0} \rightarrow \infty$, i.e., the third harmonic radiation emerging from a plasma centered about the laser focus vanishes for plasma dimensions large compared to $Z_{R}$. The conclusion that no third harmonic is generated by an infinite medium is also

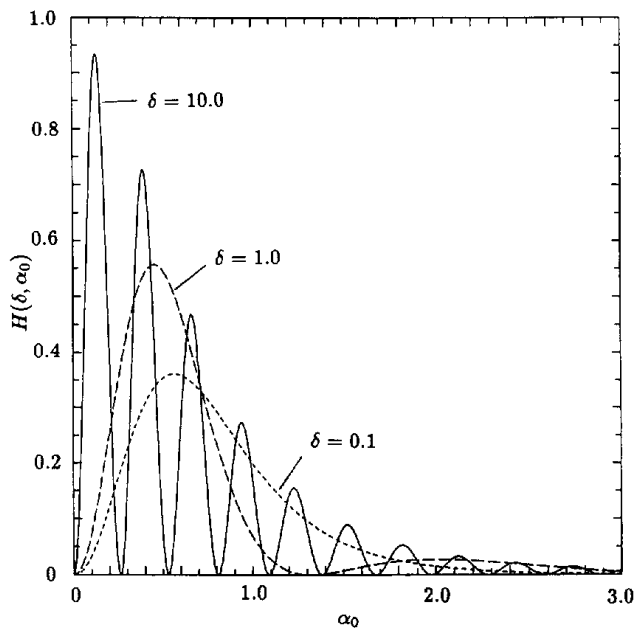

Fig. 3. The function $H\left(\delta, \alpha_{0}\right)$, proportional to the third harmonic power emerging from a plasma of width $2 z_{0}$ centered about the laser focus, verses $\alpha_{0}=z_{0} / Z_{R}$, for $\delta=2 k_{p}^{2} r_{0}^{2} / 3=10.0$ (solid curve), 1.0 (dashed curve) and 0.1 (dotted curve), where $Z_{R}=k_{0} r_{0}^{2} / 2$ is the Rayleigh length.

the case for third harmonic generation due to the nonlinear susceptibility associated with bound electrons [25].

A finite third harmonic signal, however, may be detected by focusing the pump laser on the trailing edge $(z=0)$ of a plasma slab extending from $z_{\min } \leq z \leq z_{\max }=0$, with dimensions large compared to $Z_{R}$, i.e., $z_{\min } \ll-Z_{R}$. In practice, this may be achieved by focusing the pump laser pulse on the trailing edge of a pre-ionized gas get. Approximating $\alpha_{\min }=-\infty$ and $\alpha_{\max }=0$ gives $f=f_{S}$, where

$$
\begin{gathered}
f_{S}=-\frac{k_{p}^{2} \hat{a}_{0}^{3}}{96 k_{0}^{2}}[1+g(\delta)], \\
g(\delta)=(\delta / 8)\left[1-\delta e^{\delta} E_{1}(\delta)\right] .
\end{gathered}
$$

Furthermore, it can be shown that $0 \leq 1-\delta e^{\delta} E_{1}(\delta)<$ $(1+\delta)^{-1}$. Hence, $g^{2} \ll 1$ and may be neglected. The amplitude of the third harmonic emerging from the plasma is given by $\left|a_{3}\right|=\left|f_{S}\right|\left|a_{v 3}\right|$. At the laser focus, this is a factor of $4 / 9$ smaller than that obtained from the 1-D theory in the limit $\hat{a}_{0}^{2} \ll 1$. Hence, for a semi-infinite plasma including the effects of diffraction in the limit $\hat{a}_{0}^{2} \ll 1$, the ratio of third harmonic power to the pump laser power, $P_{3} / P_{0}=3\left|f_{S}^{2}\right| / \hat{a}_{0}^{2}$, is given by

$$
P_{3} / P_{0} \simeq 3 \times 10^{-4}\left(\lambda_{0} \hat{a}_{0} / \lambda_{p}\right)^{4}
$$

which is a factor of $(4 / 9)^{2} / 3 \simeq 1 / 15$ smaller than the corresponding $1-D$ expression for the maximum power.

\section{Second Harmonic Generation}

The above results indicate that for an initially uniform plasma density, no second harmonics are excited. This is true even when the effects of a finite incident laser spot size, $r_{0}$, and the transverse gradients associated with self-consistent 3 $\mathrm{D}$ density perturbation are included, i.e., (41). Generation of even harmonics requires that the plasma have initial transverse 
density gradients prior to the arrival of the incident laser, i.e., $\nabla_{\perp} n_{0} \neq 0$, where $n_{0}(r)$ is the initial plasma density profile. Electrons undergoing quiver motion in the presence of a density gradient produce density oscillations. For a pump laser of the form $\mathbf{a}_{0}=\hat{a}_{0} \cos \theta_{0} \mathbf{e}_{x}$, the continuity equation, to first order in $\hat{a}_{0} \ll 1$, implies a density oscillation $\delta n_{1}$ of the form $\delta n_{1} \simeq\left(\hat{a}_{0} \sin \theta_{0}\right) \nabla_{x} n_{0} / k_{0}$. This produces a source current $S_{2} \simeq k_{p}^{2}\left(\delta n_{1} / n_{0}\right) \hat{a}_{0} \cos \theta_{0} \sim \sin 2 \theta_{0}$ which drives the second harmonic radiation. The amplitude of the second harmonic radiation may be estimated by approximating the density gradient by $\nabla_{x} n_{0} \simeq-n_{0} / r_{p}$, assuming $r_{p}^{2} \gg r_{0}^{2}$ and neglecting the effects of diffraction. At saturation, i.e., after a detuning length $L_{d_{2}}=2 \lambda_{p}^{2} / 3 \lambda_{0}$, the amplitude of the second harmonic is given by $\left|\hat{a}_{2}\right| \simeq \hat{a}_{0}^{2} / 3 k_{0} r_{p}$. The ratio of second harmonic power to pump laser power is $P_{2} / P_{0} \simeq$ $\left(\hat{a}_{0} \lambda_{0} / r_{p}\right)^{2} \%$ and, hence, $P_{2} \sim I_{0}^{2}$. Not only do initial density gradients lead to the generation of even harmonics for linearly polarized incident lasers, it also implies that circularly polarized incident lasers will generate harmonics, both even and odd.

Transverse plasma density gradients can occur when a Gaussian laser pulse produces intensity-dependent ionization of a neutral gas. For laser pulses with peak intensities $I_{0}>I_{S}$ (where $I_{S}$ is the saturation intensity for which the gas is fully ionized) ionizing a uniform gas, density gradients will exist in the "halo" region about the laser focus. This halo region is the portion of the interaction region in which the gas is not fully ionized and corresponds approximately to focal regions in which the laser intensity lies within the band $I_{\min }<I<I_{S}$ (where $I_{\min }$ is the minimum intensity required to produce ionization). As the peak intensity, $I_{0}$, increases, one can show that the volume of this halo region increases as $I_{0}^{3 / 2}$, where a Gaussian laser pulse of the form given by (35) has been assumed (i.e., a spherical lens and a double cone focal geometry) [26]. Second harmonics will be produced from the halo region. The intensity scaling of the second harmonic power with peak intensity will tend to be dominated by effects associated with the increasing volume of the halo region. Hence, for $I_{0}>I_{S}$, the volume effect implies a second harmonic power scaling of $P_{2} \sim I_{0}^{3 / 2}$. Furthermore, in the partially ionized halo region, the production of harmonic radiation can be significantly enhanced and/or dominated by atomic [13] and ionization [14] processes [17], [18]. In long-pulse laser-plasma experiments, filamentation may be a dominant mechanism in second harmonic generation [15], [27].

\section{CONCLUSION}

A nonlinear cold fluid model valid in the regime $\hat{a}_{0}^{2} \geq 1$, given by (10) and (11), has been formulated and used to analyze relativistic harmonic generation. The self-consistent collective plasma response is included and shown to significantly reduce the source current driving the harmonic radiation. A general expression for the nonlinear index of refraction was derived (12), and is a function of only the electrostatic (space-charge) potential of the plasma, $\phi$. The slow part of the index of refraction determined the dispersion relation (13) and the fast part determined the source current for the harmonics. Saturation of the $N^{\text {th }}$ harmonic occurs after a detuning length, $L_{d_{N}}=c \tau_{d_{N}}$, given by (30). The harmonic amplitude is maximum when the laser-plasma interaction length, $L$, is an integer multiple of $L_{d_{N}}$. The ratio of saturated power in the $N^{\text {th }}$ harmonic to that in the incident laser is given by (34). This expression is valid for long lasers pulses, $c \tau_{L} \gg \lambda_{p}\left(n_{0} \gg 10^{16} \mathrm{~cm}^{-3}\right.$ for $\left.\tau_{L} \simeq 1 \mathrm{psec}\right)$, and for interaction distances short compared to the diffraction length, $L \ll \pi Z_{R}$. Relativistic harmonic generation favors the use of high densities and intense lasers, $\hat{a}_{0} \sim 1$. The saturated power given by (34), in the limit $\hat{a}_{0}^{2} \ll 1$, is a factor of $b_{N}\left(\lambda_{0} / \lambda_{p}\right)^{2(N-1)}$ smaller, where $b_{N}$ are constants, than that predicted by the simplified quiver model, which neglects the self-consistent plasma response. This reduction in the harmonic power by collective plasma effects is supported by recent experiments on harmonic generation in pre-ionized plasmas [2], [17], [18].

The effects of a diffracting incident laser field with a finite spot size $r_{0}$ have been analyzed in the limit $\hat{a}_{0}^{2} \ll$ 1. Diffraction is important for interaction lengths $L \gtrsim \pi Z_{R}$ $\left(L_{d_{N}} \geq \pi Z_{R}\right.$ implies $\delta=2 k_{p}^{2} r_{0}^{2} / 3 \leq 1$ for the third harmonic). It is shown that no third harmonic signal emerges from a plasma of near infinite extent. A finite third harmonic signal requires the use of a semi-infinite or finite slab plasma. The third harmonic power emerging from the edge of a semiinfinite plasma, which corresponds to the focal point of the incident laser, is given by (55) and is a factor of 15 smaller than the corresponding 1-D saturation power. No second harmonics are generated from an initially uniform plasma. The generation of even harmonics requires the existence of transverse gradients in the initial plasma density. Circularly polarized light will also generate both even and odd harmonics when initial transverse density gradients are present.

The most severe constraint on the production of coherent relativistic harmonic radiation is that of phase detuning. In a dense plasma with $n_{0}=10^{20} \mathrm{~cm}^{-3}\left(\lambda_{p}=3.4 \mu \mathrm{m}\right)$ and a $\lambda_{0}=1 \mu \mathrm{m}$ laser with $I_{0}=6.7 \times 10^{18} \mathrm{~W} / \mathrm{cm}^{2}\left(\hat{a}_{0}=2.2\right)$, the saturation efficiencies for the third and fifth harmonics are $P_{3} / P_{0}=2.2 \times 10^{-5}$ and $P_{5} / P_{0}=4.6 \times 10^{-10}$, respectively. At such a high density the detuning lengths are extremely short, $L_{d_{3}}=8.0 \mu \mathrm{m}$ for the third harmonic and $L_{d_{5}}=4.3 \mu \mathrm{m}$ for the fifth harmonic. If a scheme for phase matching could be conceived, the interaction distance, $L$, and, hence, the harmonic power, $P_{N} \sim L^{2}$, could be dramatically increased.

\section{ACKNOWLEDGMENT}

The authors thank T. Swyden for his numerical assistance and $\mathrm{W}$. Mori for useful discussions.

\section{REFERENCES}

[1] P. Sprangle and E. Esarey, "Interaction of ultrahigh laser fields with beams and plasmas," Phys. Fluids B, vol. 4, pp. 2241-2248, 1992

[2] G. Mourou and D. Umstadter, "Development and applications of compact high-intensity lasers," Phys. Fluids B, vol. 4, pp. 2315-2325, 1992.

[3] C. Max, J. Arons and A. B. Langdon, Self-modulation and self-focusing of electromagnetic waves in plasmas," Phys. Rev. Lett., vol. 33, pp. 209-212, 1974; G. Z. Sun, E. Ott, Y. C. Lee, and P. Guzdar, "Selffocusing of short intense pulses in plasmas," Phys. Fluids, vol. 30, pp. 
526-532, 1987; W. B. Mori, C. Joshi, J. M. Dawson, D. W. Forslund, and I. M. Kindel, "Evolution of self-focusing of intense electromagnetic waves in plasma," Phys. Rev. Lett., vol. 60, pp. 1298-1301, 1988.

[4] P. Sprangle, C. M. Tang, and E. Esarey, "Relativistic self-focusing of short-pulse radiation beams in plasmas," IEEE Trans. Plasma Sci., vol. PS-15, pp. 145-153, 1987. E. Esarey, A. Ting, and P. Sprangle, "Relativistic focusing and beat wave phase velocity control in the plasma beat wave accelerator," Appl. Phys. Lett., vol. 53, pp. 1266-1268, 1988.

[5] T. Tajima and J. M. Dawson, "Laser electron accelerator," Phys. Rev. Lett., vol. 43, pp. 267-270, 1979; L. M. Gorbunov and V. I. Kirsanov, "Excitation of plasma waves by an electromagnetic wave packet," $Z h$. Eksp. Teor. Fiz., vol. 93, pp. 509-518, 1987 [Sov. Phys. JETP, vol. 66, pp. 290-294, 1987].

[6] P. Sprangle, E. Esarey, A. Ting, and G. Joyce, "Laser wakefield acceleration and relativistic optical guiding," Appl. Phys. Lett., vol. 53, pp. 2146-2148, 1988; E. Esarey, A. Ting, P. Sprangle, and G. Joyce, "The laser wakefield accelerator," Comments Plasma Phys. Controlled Fusion, vol. 12, pp. 191-204, 1989.

[7] A. Ting, P. Sprangle, and E. Esarey, "Simulation of short intense laser pulse propagation in the laser wakefield accelerator," in Advanced Accelerator Concepts, AIP Conf. Proc. 193, C. Joshi, Ed., New York: AIP, 1989, pp. 398-407.

[8] P. Sprangle, E. Esarey, and A. Ting, "Nonlinear theory of intense laserplasma interactions," Phys. Rev. Lett., vol. 64, pp. 2011-2014, 1990; "Nonlinear interaction of intense laser pulses in plasmas," Phys. Rev. A, vol. 41 , pp. $4463-4469,1990$.

[9] A. Ting, E. Esarey, and P. Sprangle, "Nonlinear wakefield generation and relativistic focusing of intense laser pulses in plasmas," Phys. Fluids B, vol. 2, pp. 1390-1394, 1990.

[10] P. Sprangle, E. Esarey, J. Krall, and G. Joyce, "Propagation and guiding of intense laser pulses in plasma," Phys. Rev. Lett., vol. 69, pp. 2200-2203, 1992.

[11] W. M. Wood, G. Focht, and M. C. Downer, "Tight focusing and blue shifting of millijoule femtosecond pulses from a conical axicon amplifier", Opt. Lett., vol. 13, pp. 984-986, 1988; S. C. Wilks, J. M. Dawson, W. B. Mori, T. Katsouleas, and M. E. Jones, "Photon accelerator," Phys. Rev. Lett., vol. 62, pp. 2600-2603, 1989; H. C. Kapteyn and M. M. Murnane, "Relativistic pulse compression," J. Opt. Soc. Am. B, vol. 8, pp. 1657-1662, 1991; W. B. Mori, "Generation of tunable radiation using an underdense ionization front," Phys. Rev. A, vol. 44, pp. 5118-5121, 1991.

[12] E. Esarey, A. Ting, and P. Sprangle, "Frequency shifts induced in laser pulses by plasma waves," Phys. Rev. A, vol. 42, pp. 3526-3531, 1990; E. Esarey, G. Joyce, and P. Sprangle, "Frequency up-shifting of laser pulses by copropagating ionization fronts," Phys. Rev. A, vol. 44, pp. 3908-3911, 1991.

[13] A. McPherson, G. Gibson, A. Jarah, U. Johann, T. S. Luk, F. McIntyre, K. Boyer, and C. Rhodes, "Studies of multiphoton production of vacuum-ultraviolet radiation in the rare gases", J. Opt. Soc. Am. B, vol. 4, 595-601, 1987; M. Ferray, A. L'Huillier, X. F. Li, L. A. Lompre', G. Mainfray, and C. Manus, "Multiple-harmonic conversion of 1064 nm radiation in rare gases", $J$. Phys. $B$, vol. 21, L31-L35, 1988; L. A. Lompre', A. L'Huillier, and G. Mainfray, "Toward extreme VUV by harmonic generation in strong fields," in Coherent Short Wavelength Radiation: Generation and Application, vol. 11, P. Bucksbaum and N. Ceglio, Eds., Washington, DC: Opt. Soc. Amer., 1991, pp. 2-6; M. Perry, et al., submitted to this issue.

[14] F. Brunel, "Harmonic generation due to plasma effects in a gas undergoing multiphoton ionization in the high-intensity limit," J. Opt. Soc. Am. B, vol. 7, pp. 521-526, 1990.

[15] W. P. Leemans, C. E. Clayton, W. B. Mori, K. A. Marsh, A. Dyson, and C. Joshi, "Plasma physics aspects of tunnel-ionized gases," Phys. Rev. Lett., vol. 68, pp. 321-324, 1992; W. P. Leemans, C. E. Clayton, W. B. Mori, K. A. Marsh, P. K. Kaw, A. Dyson, C. Joshi, and J. M. Wallace, "Experiments and simulations of tunnel-ionized plasmas," Phys. Rev. A, vol. 46, pp. 1091-1105, 1992.

[16] C. B. Darrow, C. Coverdale, M. D. Perry, W. B. Mori, C. Clayton, K.
Marsh and C. Joshi, "Strongly coupled stimulated Raman backscatter from subpicosecond laser-plasma interactions," Phys. Rev. Lett., vol. 69, pp. 442-445, 1992.

[17] X. Liu, J. S. Coe, C. Y. Chien, D. Umstadter, E. Esarey, and P. Sprangle, "Harmonic generation by an intense picosecond laser in an underdense plasma," in Coherent Short Wavelength Radiation: Generation and Application, vol. 11, P. Bucksbaum and N. Ceglio, Eds., Washington, DC: Opt. Soc. Amer., 1991, pp. 7-11.

[18] X. Liu, D. Umstadter, A. Ting and E. Esarey, submitted to this issue.

[19] W. B. Mori, et al., submitted to this issue.

[20] J. M. Rax and N. J. Fisch, "Third-harmonic generation with ultrahighintensity laser pulses," Phys. Rev. Lett., vol. 69, pp. 772-775, 1992; J. M. Rax, et al., submitted to this issue.

[21] P. Sprangle and E. Esarey, "Stimulated backscattered harmonic generation from intense laser interactions with beams and plasmas," Phys. Rev. Lett., vol. 67, pp. 2021-2024, 1991; E. Esarey and P. Sprangle, "Generation of stimulated backscattered harmonic radiation from intense laser interactions with beams and plasmas," Phys. Rev. A, vol. 45, pp. 5872-5882, 1992.

[22] E. S. Sarachik and G. T. Schappert, "Classical theory of the scattering of intense laser radiation by free electrons," Phys. Rev. D, vol. 1, pp. 2738-2753, 1970

[23] D. Strickland and G. Mourou, "Compression of amplified chirped optical pulses," Opt. Commun., vol. 56, pp. 219-221, 1985; P. Maine, D. Strickland, P. Bado, M. Pessot, and G. Mourou, "Generation of ultrahigh peak power pulses by chirped-pulse amplification," IEEE J. Quantum Electron., vol. QE-24, pp. 398-403, 1988; M. D. Perry. F. G. Patterson, and J. Weston, "Spectral shaping in chirped pulse amplification," Opt. Lett., vol. 15, pp. 381-383, 1990; F. G. Patterson, and M. Perry, "Design and performance of a multiterawatt, subpicosecond Nd:glass laser," $J$. Opt. Soc. Am. B, vol. 8, pp. 2384-2391, 1991.

[24] A. Yariv, Quantum Electronics, Third Edition, New York: Wiley, 1989, p. 118.

[25] J. F. Ward and G. H. C. New, "Optical third harmonic generation in gases by a focused laser beam," Phys. Rev., vol. 185, pp. 57-72, 1969.

[26] M. R. Cervenan, R. H. C. Chan and N. R. Isenor, "Three photon ionization of alkali atoms at the ruby laser wavelength," Can. J. Phys., vol. 53, pp. 1573-1578, 1975.

[27] J. Meyer and Y. Zhu, "Second harmonic emission from an underdense laser-produced plasma and filamentation," Phys. Fluids, vol. 30, pp. 890-895, 1987.

E. Esarey, photograph and biography not available at the time of publication.

A. Ting, photograph and biography not available at the time of publication.

P. Sprangle, photograph and biography not available at the time of publication.

D. Umstadter, photograph and biography not available at the time of publication.

X. Liu, photograph and biography not available at the time of publication. 